Discrete Comput Geom 30:373-377 (2003)

DOI: $10.1007 / \mathrm{s} 00454-003-2823-y$

\title{
The Density of Shapes in Three-Dimensional Barycentric Subdivision*
}

\author{
Richard Evan Schwartz \\ Department of Mathematics, University of Maryland, \\ College Park, MD 20742-4015, USA \\ res@math.umd.edu
}

\begin{abstract}
We prove that the infinite process of iterated barycentric subdivision, when applied to a tetrahedron, produces a dense set of shapes of smaller tetrahedra.
\end{abstract}

\section{Introduction}

The barycentric subdivision of an $n$-dimensional simplex $\Delta$ is a certain collection of $(n+1)$ ! smaller $n$-simplices whose union is $\Delta$. The construction is defined by induction on $n$. If $n=0$, then $\Delta$ is a single point, and the barycentric subdivision of $\Delta$ is this same point. In general, if $\Delta^{\prime}$ is one of the simplices in the barycentric subdivision of $\Delta$, then $\Delta^{\prime}$ is the convex hull of a set of the form $v \cup F^{\prime}$, where $v$ is the center of mass of $\Delta$-i.e., the barycenter-and $F^{\prime}$ is one of the simplices in the barycentric subdivision of one of the top dimensional faces $F$ of $\Delta$. See p. 123 of [S] or Section 2 below for more details.

Consider the following dynamical process: Start with an $n$-simplex $\Delta$ and barycentrically subdivide $\Delta$ into simplices $\Delta_{1}, \ldots, \Delta_{(n+1) !}$. Next, subdivide $\Delta_{j}$ into simplices $\Delta_{j 1}, \ldots, \Delta_{j(n+1) !}$, for each $j$, and so forth. This process produces an infinite collection $C$ of simplices. A natural question is: Does $C$ consist of a dense set of shapes? By shape we mean a simplex modulo similarities.

In $[\mathrm{BBC}]$ this question was raised and answered in the two-dimensional case. Part of the idea works in all dimensions. Let $\mathcal{T}$ be the collection of matrices of the form $T=L /|\operatorname{det}(L)|^{1 / n}$, where $L$ is the linear part of an affine map from $\Delta$ to a member of $C$. The affine naturality of barycentric subdivision forces $\mathcal{T}$ to be a semigroup of $S L_{n}(\boldsymbol{R})$, the group of $n \times n$ determinant-1 matrices.

When $n=2$, a calculation in [BBC] shows that $\mathcal{T}$ contains some infinite-order elliptic elements. (In general, an elliptic element of $S L_{n}(\boldsymbol{R})$ is a matrix which generates

* This research was supported by N.S.F. Research Grant DMS-0072607. 
a subgroup having compact closure, which happens iff the matrix is diagonalizable over $\boldsymbol{C}$ with all eigenvalues unit complex numbers.) The set of powers of an infinite-order elliptic element is dense in a compact subgroup of $S L_{2}(\boldsymbol{R})$ and these dense sets are used to show that $\mathcal{T}$ is dense in $S L_{2}(\boldsymbol{R})$. Hence, in the two-dimensional case, $C$ contains a dense set of triangles.

Using a computer search, which we detail in the next section, we found some infiniteorder elliptic elements in the three-dimensional case. This seems like a lucky accident, because the set of elliptic elements in $S L_{n}(\boldsymbol{R})$ has measure zero for $n \geq 3$. Using these elliptic elements, some basic Lie group theory, and Mathematica [W], we prove

Theorem 1.1. The three-dimensional barycentric subdivision process produces a dense set of shapes of tetrahedra.

A similar computer search failed to turn up any elliptic elements in the case $n=4$, though we certainly would have liked to make a deeper search using a more powerful computer. We think that the density result should be true in all dimensions, whether or not $\mathcal{T}$ contains elliptic elements.

\section{The Proof}

Here we give a concrete description of barycentric subdivision in the three-dimensional case. Let $\Delta$ be the convex hull of points $v_{0}, v_{1}, v_{2}, v_{3} \in \boldsymbol{R}^{3}$. Let $S_{4}$ be the group of permutations of the set $\{0,1,2,3\}$. Given $\sigma=\left(i_{0}, i_{1}, i_{2}, i_{3}\right) \in S_{4}$, let $c_{k}$ be the center of mass of the points $v_{i_{0}}, \ldots, v_{i_{k}}$. Let $\Delta_{\sigma}$ be the convex hull of the points $c_{0}, c_{1}, c_{2}, c_{3}$. The union $\bigcup_{\sigma \in S_{4}} \Delta_{\sigma}$ is the barycentric subdivision of $\Delta$.

To begin our dynamical process, we take the initial tetrahedron $\Delta$ to be the convex hull of the vertices $e_{0}, e_{1}, e_{2}, e_{3}$. Here $e_{0}$ is the origin and $\left\{e_{1}, e_{2}, e_{3}\right\}$ is the standard basis of $\boldsymbol{R}^{3}$. Let $A_{\sigma}$ be the affine map such that $A_{\sigma}\left(e_{k}\right)=c_{k}$ for $k=0,1,2,3$. Let $L_{\sigma}$ be the linear part of $A_{\sigma}$. Finally, let $T_{\sigma}=L_{\sigma} /\left|\operatorname{det}\left(L_{\sigma}\right)\right|^{1 / 3}$. By construction, $A_{\sigma}(\Delta)=\Delta_{\sigma}$ and therefore $T_{\sigma} \subset \mathcal{T}$, the semigroup discussed in Section 1 .

We order the 24 elements of $S_{4}$ lexicographically. For instance, $\sigma_{1}=(0123)$ and $\sigma_{2}=(0132)$. We define

$$
F(i, j, k)=T_{\sigma_{k}} \circ T_{\sigma_{j}} \circ T_{\sigma_{i}} .
$$

Say that the triple $(i, j, k)$ is good if $F(i, j, k)$ is an infinite-order elliptic element. A computer search reveals 39 good sequences. Here is the list, modulo cyclic permutations:

$(2,15,19) ;(5,8,23) ;(5,19,18) ;(5,20,16) ;(7,17,8) ;(8,18,9) ;(8,18,20)$;

$(8,23,16) ;(9,19,23) ;(15,19,16) ;(16,16,19) ;(16,19,18) ;(19,23,20)$.

We had hoped to see a divine pattern in this list, but did not.

Our density proof uses only the elements

$$
S=F(23,20,19) ; \quad M_{1}=F(5,20,16) ; \quad M_{2}=F(20,16,5) .
$$

Another triple of elements from the list would probably work just as well. In the Appendix 
we include a short Mathematica program which computes

$$
\begin{aligned}
& S=\frac{1}{24}\left[\begin{array}{ccc}
54 & 48 & 39 \\
-6 & -32 & -35 \\
-78 & -32 & -23
\end{array}\right] ; \quad M_{1}=\frac{1}{72}\left[\begin{array}{ccc}
-60 & -68 & -27 \\
36 & 12 & 81 \\
-60 & 4 & 27
\end{array}\right] \\
& M_{2}=\frac{1}{24}\left[\begin{array}{ccc}
18 & 12 & 21 \\
-54 & -68 & -71 \\
54 & 52 & 43
\end{array}\right] \text {. }
\end{aligned}
$$

Lemma 2.1. $S, M_{1}$, and $M_{2}$ are infinite-order elliptic elements of $S L_{3}(\boldsymbol{R})$.

Proof. The eigenvalues of $S$ and $M_{j}$ respectively are $\{1, \alpha, \bar{\alpha}\}$ and $\{1, \beta, \bar{\beta}\}$, where $\alpha=-25 / 48+i \sqrt{1679} / 48$ and $\beta=-31 / 48+i \sqrt{1343} / 48$. Both $\alpha$ and $\beta$ have norm 1 , so $S$ and $M_{j}$ are elliptic. If $S$ had finite order, then $\alpha$ would be a primitive $n$th root of unity for some $n$. Then $\alpha$ would have $\varphi(n)$ distinct Galois conjugates, where $\varphi$ is the Euler phi-function. Since $\alpha$ is a quadratic irrational, we have $\varphi(n)=2$. The forces $n \leq 6$. Clearly, $\alpha$ is not an $n$th root of unity for $n \leq 6$. Hence $S$ has infinite order. The same argument works for $M_{j}$.

Let $\langle S\rangle$ be the closure of the semigroup generated by $S$. Since $S$ is infinite-order elliptic, $\langle S\rangle$ is a closed one-parameter compact subgroup. Let $G \subset S L_{3}(\boldsymbol{R})$ be the closed subgroup generated by the eight compact subgroups $G_{i j}=M_{i}^{j}\langle S\rangle M_{i}^{-j}$. Here $i \in\{1,2\}$ and $j \in\{1,2,3,4\}$.

Lemma 2.2. $G=S L_{3}(\boldsymbol{R})$.

Proof. The lie algebra to $S L_{3}(\boldsymbol{R})$ is $\mathfrak{s l}_{3}(\boldsymbol{R})$, the space of traceless $3 \times 3$ matrices. Below we justify the claim that

$$
\mathfrak{s}=\left[\begin{array}{ccc}
70 & 54 & 57 \\
-114 & -107 & -104 \\
18 & 52 & 37
\end{array}\right] \in \mathfrak{s l}_{3}(\boldsymbol{R})
$$

generates $\langle S\rangle$. By this we mean that

$$
\langle S\rangle=\{\exp (t \mathfrak{s}) \mid t \in \boldsymbol{R}\}
$$

For $i$ and $j$ as above we define $\mathfrak{g}_{i j}=M_{i}^{j} \mathfrak{s} M_{i}^{-j}$. By construction

$$
G_{i j}=\left\{\exp \left(t \mathfrak{g}_{i j}\right) \mid t \in \boldsymbol{R}\right\} .
$$

Let $\mathfrak{G}$ be the vector space spanned by the eight vectors $\mathfrak{g}_{i j}$.

For any lie algebra vectors $\mathfrak{a}$ and $\mathfrak{b}$ we have the well-known formula

$$
\exp (\mathfrak{a}+\mathfrak{b})=\lim _{k \rightarrow \infty}\left(\exp \left(\frac{\mathfrak{a}}{k}\right) \cdot \exp \left(\frac{\mathfrak{b}}{k}\right)\right)^{k}
$$

(See Exercise 8.38 of $[\mathrm{FH}]$.) This formula easily implies that $\exp (\mathfrak{G}) \subset G$. Since $\operatorname{dim}\left(\mathfrak{s l}_{3}(\boldsymbol{R})\right)=8$, all we need to prove is that $\operatorname{dim}(\mathfrak{G})=8$. There is a natural map 
$P: \mathfrak{s l}_{3}(\boldsymbol{R}) \rightarrow \boldsymbol{R}^{8}$. We simply string out the coordinates of a trace-zero matrix $\mathfrak{g}$, leaving off $\mathfrak{g}(3,3)$. It is easy to see that $P$ is a vector space isomorphism. Let $M$ be the $8 \times 8$ matrix whose rows are $P\left(\mathfrak{g}_{i j}\right)$. We compute

$$
\operatorname{det}(M)=\frac{1574679337686718881331462994390117}{159739999685311463424} \neq 0 .
$$

This is only possible if the vectors $P\left(\mathfrak{g}_{i j}\right)$ span $\boldsymbol{R}^{8}$.

Let $\overline{\mathcal{T}}$ be the closure of $\mathcal{T}$ in $S L_{3}(\boldsymbol{R})$. By construction $\langle S\rangle \subset \overline{\mathcal{T}}$. Since $M_{j}$ is an infinite-order elliptic element, $M_{i}^{ \pm j} \in \overline{\mathcal{T}}$ for all relevant $i$ and $j$. Therefore the group $G_{i j}$ is contained in the semigroup $\overline{\mathcal{T}}$. This implies that $G \subset \overline{\mathcal{T}}$. However, $G=S L_{3}(\boldsymbol{R})$. Therefore $\mathcal{T}$ is dense in $S L_{3}(\boldsymbol{R})$. Our theorem follows immediately from this.

Our only piece of unfinished business is to justify the formula for $\mathfrak{s}$. By computing the eigenspaces of $S$ we find that the matrix

$$
U=\left[\begin{array}{ccc}
-21 & 0 & 2 \\
-34 & -1 & -3 \\
58 & 2 & 0
\end{array}\right]
$$

conjugates $S$ to block triangular form:

$$
U^{-1} S U=\left[\begin{array}{ll}
1 & 0 \\
0 & B
\end{array}\right] ; \quad B=\frac{1}{48}\left[\begin{array}{cc}
-14 & -60 \\
30 & -36
\end{array}\right] .
$$

Note that $B \in S L_{2}(\boldsymbol{R})$ is infinite-order elliptic. Let $\langle B\rangle$ be the closure of the group generated by $B$. We claim that the matrix

$$
\mathfrak{b}=48 B-24 \operatorname{trace}(B) I=\left[\begin{array}{ll}
11 & -60 \\
30 & -11
\end{array}\right] \in \mathfrak{s l}_{2}(\boldsymbol{R})
$$

generates $\langle B\rangle$ in the sense that $\langle B\rangle=\{\exp (t \mathfrak{b}) \mid t \in \boldsymbol{R}\}$. To prove this, we note that $\mathfrak{b}$ and $B$ commute, when multiplied together as matrices. Hence, for any $t \in \boldsymbol{R}$ the element $\beta_{t}=\exp (t \mathfrak{b})$ commutes with any element of $\langle B\rangle$. As is well known $S L_{2}(\boldsymbol{R})$ acts isometrically on the hyperbolic plane $\boldsymbol{H}^{2}$ by linear fractional transformations. The group $\langle B\rangle$, which consists entirely of elliptic elements, acts as the group of isometric rotations about some fixed point $x \in \boldsymbol{H}^{2}$. Since $\beta_{t}$ commutes with all elements of $\langle B\rangle$, it must also act as an isometric rotation about $x$. Hence $\beta_{t} \subset\langle B\rangle$ for all $t$. Our claim now follows easily.

Since $\mathfrak{b}$ generates $\langle B\rangle$,

$$
\mathfrak{s}=U\left[\begin{array}{ll}
0 & 0 \\
0 & \mathfrak{b}
\end{array}\right] U^{-1}
$$

generates $\langle S\rangle$ in the sense of Lemma 2.1. Expanding this product gives the formula for $\mathfrak{s}$ used in Lemma 2.1.

\section{Acknowledgment}

I thank Bill Goldman for some interesting discussions about Lie groups and Lie algebras. 


\section{Appendix. A Mathematica File}

We refer the reader to [W] for details on the implementation of Mathematica. A copy of this file produced our calculations.

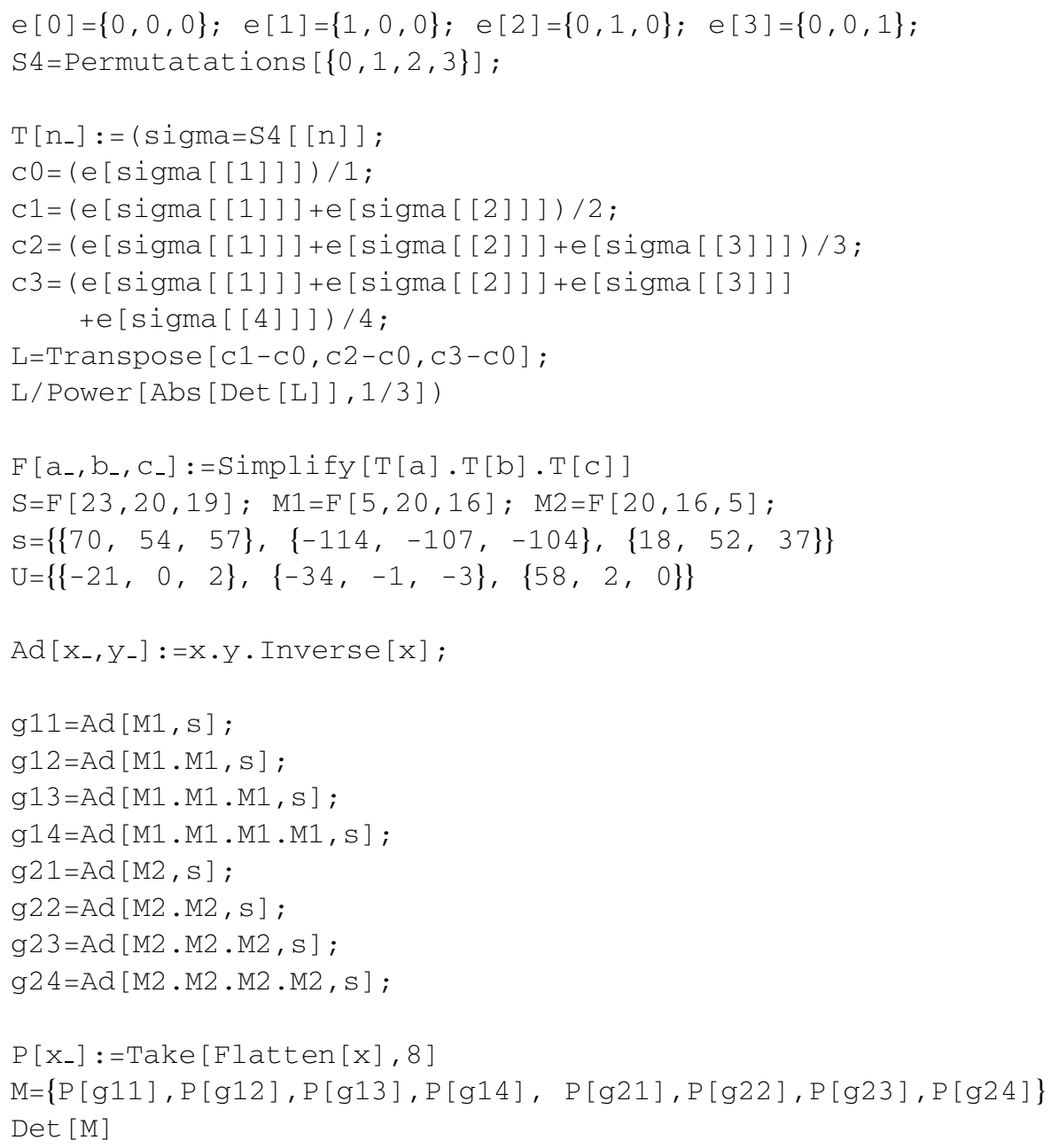

\section{References}

[BBC] I. Bárány, A.F. Beardon, and T.K. Carne, Barycentric subdivision of triangles and semigroups of Mobius maps, Mathematika 43 (1996).

[FH] W. Fulton and J. Harris, Representation Theory, A First Course, GTM 129, Springer-Verlag, New York, 1991.

[S] E. Spanier, Algebraic Topology, Springer-Verlag, New York, 1966.

[W] S. Wolfram, The Mathematica Book, fourth edition, Cambridge University Press, Cambridge, 1999. 